Kansas State University Libraries

New Prairie Press

\title{
USING NONLINEAR FIXED AND MIXED MODELS TO STUDY ACCLIMATION TO HEAT STRESS IN CATTLE
}

L. Lan
A. M. Parkhurst
D. A. Spiers
K. M. Eskridge
G. L. Hahn

See next page for additional authors

Follow this and additional works at: https://newprairiepress.org/agstatconference

Part of the Agriculture Commons, and the Applied Statistics Commons

\section{(c) (1) $\Theta($}

This work is licensed under a Creative Commons Attribution-Noncommercial-No Derivative Works 4.0 License.

\section{Recommended Citation}

Lan, L.; Parkhurst, A. M.; Spiers, D. A.; Eskridge, K. M.; and Hahn, G. L. (2002). "USING NONLINEAR FIXED AND MIXED MODELS TO STUDY ACCLIMATION TO HEAT STRESS IN CATTLE," Conference on Applied Statistics in Agriculture. https://doi.org/10.4148/2475-7772.1207

This is brought to you for free and open access by the Conferences at New Prairie Press. It has been accepted for inclusion in Conference on Applied Statistics in Agriculture by an authorized administrator of New Prairie Press. For more information, please contact cads@k-state.edu. 


\section{Author Information}

L. Lan, A. M. Parkhurst, D. A. Spiers, K. M. Eskridge, and G. L. Hahn 


\title{
Using Nonlinear Fixed and Mixed Models to Study Acclimation to Heat Stress in Cattle
}

\author{
L. Lan ${ }^{1}$, A. M. Parkhurst ${ }^{1}$, D. A.Spiers ${ }^{2}$, K. M. Eskridge ${ }^{1}$, and G. L. Hahn ${ }^{3}$ \\ ${ }^{1}$ University of Nebraska - Lincoln \\ ${ }^{2}$ University of Missouri - Columbia \\ ${ }^{3}$ U.S. Meat Animal Research Center, U.S. Department of Agriculture
}

\begin{abstract}
Researchers studying acclimation of cattle to heat stress want to know if exposure to heat stress in controlled chambers will help cattle adjust to climatic conditions in the field. The four parameter nonlinear PET model is used to study the relationship between core body temperature and ambient temperature. This model works well when cattle are challenged by heat stress but the model is less useful for thermoneutral conditions. Both proc Nlin and Nlmixed are used to compare and contrast the field parameters between the controlled and the potentially acclimated group. Simulation studies were used to compare the effectiveness of proc Nlin versus proc NImixed. The results are helpful, not only for researchers who study acclimation, but also for those who study sensitivity, tolerance and robustness of cattle during heat stress.
\end{abstract}

Key Words: Acclimation, PET model, Nlin, Nlinmixed

\section{Introduction}

The heat stress environmental temperature influences the health and performance of steers greatly and may even result in economical loss. To reduce the impact of hot weather on cattle, we study acclimation. Heat stress has been described as the delay in animal body temperature caused by hot environmental temperature (Hahn, 1989). Acclimation can then be identified as the decrease in lag or delay as the animal's body temperature adjusts to the heat challenge.

A nonlinear model developed by our group, termed the extended-PET-period-two model describes animal body temperature, $\mathrm{T}_{\mathrm{b}}$ as a function of the ambient temperature, $\mathrm{T}_{\mathrm{a}}$ (Feng et.al, 2001). This model assumes Ta is the principle driving force for $T_{b}$ (Hahn, 1989). In other words, the model holds when the steers are challenged by exposure to hot environmental temperature. All 4 parameters $\left(\kappa, \delta, \gamma, T_{\text {Binitial }}\right)$ in this model have biological meaning. But the most important parameter to measure acclimation is a function of $\kappa$ called $\tau$. The parameter $\tau$ is the lag of $T_{b}$ with respect to $T_{a}$,

Historically, treatment differences in nonlinear models have been studied by estimating the parameters for each steer and performing an ANOVA separately for each parameter (Seber and Wild,1989). Another approach is to consider the steers under the same treatment to be from the same population. This is the fixed effect nonlinear model. There is one set of parameter estimates for all the steers in each treatment population. Finally, a third approach is now readily available. The nonlinear mixed model takes into account the random effect of an individual steer within the same population. Quite possibly the individual behavior of steers is different even though they receive the same treatment. Thus the mixed effect model should be more appropriate than the fixed effect model. 
The experimental data were analyzed by each approach and conclusions were drawn. Next a simulation study was conducted to evaluate the effectiveness of the three approaches. The first question is: Do these approaches give similar results so that conclusions about acclimation are consistent? The second question is: How do the approaches compare as error estimates similar to those suggested by the experimental data vary?

\section{Model Equation and Parameters}

The extended-PET-period-two model is used to describe Ta and Tb with respect to the time( Parkhurst et.al, 1999; Parkhurst et.al, 2000; Feng et.al, 2001).

First, $\mathrm{T}_{\mathrm{a}}$ is modeled as a sinusoidal function with two periods:

$$
T_{a}=u_{a}+\sum_{i=1}^{2} a_{i} \sin \left[i \omega\left(t-\Phi_{i}\right)\right]
$$

Then, by Newton's law of cooling, we assume the heat stress, $\mathrm{T}_{\mathrm{a}}$, is the principle driving force to $T_{b}$. Thus, $T_{b}$ can be solved as:

$$
T_{b}=e^{-\kappa l}\left[T_{b 0}+\kappa\left(S_{0}+S_{1}\right)\right]
$$

where $S_{0}=\left(\gamma \mu_{a}+\Delta\right)\left(e^{\kappa l}-1\right) / \kappa$

and $\quad S_{1}=\gamma \sum_{i=1}^{2} \frac{\alpha_{i}}{\sqrt{\kappa^{2}+(i \omega)^{2}}}\left[e^{\kappa t} \sin \left(i \omega\left(t-\phi_{i}\right)-\theta_{i}\right)+\sin \left(\omega \phi_{i}+\theta_{i}\right)\right]$

The parameters in model II are defined as:

$\kappa:$ a thermal constant per unit time. It represents how rapidly $T_{b}$ adjusts to changes in $T_{a}$. The unit is $1 /$ hour

$\delta$ : the gradient parameter, is the difference between $T_{b}$ and the adjusted $T_{a}$

$$
\Delta=\mathrm{T}_{\mathrm{b}}-\gamma \mathrm{T}_{\mathrm{a}}
$$

$\gamma$ : the proportion of variance in $T_{b}$ comparable to variance in $T_{a}$

$$
\gamma=\frac{\sigma_{B}^{2}}{\sigma_{a}^{2}}
$$

$\mathrm{T}_{\mathrm{b} 0}$ : the steer body temperature at time zero

Frequently, it is helpful to convert $\kappa$ to the lag time.

$\tau$ : the lag of $T_{b}$ with respect to $T_{a}$, is a measure of how long it takes an animal to respond to $T_{a}$.

$$
\tau=\frac{\tan ^{-1}\left(\frac{\omega}{\kappa}\right)}{\omega}
$$

Figure 1 is an example of the delay or lag. 


\section{Motivating Experiment}

An experiment to study the acclimation of steers to heat stress was conducted by a group of researchers led by Dr. Donald Spiers in the summer of 1999 at the University of MissouriColumbia. Twelve crossbred Bos Taurus steers were placed in two different chambers where the ambient temperature was controlled roughly as a sinusoidal process. The experiment lasted for 24 days (from May $7^{\text {th }}$ to May $31^{\text {st }}$, 1999). Six steers were randomly placed in the control (thermoneutral) group. They were housed in chamber A where the ambient temperature, Ta, was controlled to be $19 \pm 7 \mathrm{C}$ for all 24 days. Another six steers were assigned to the potentially acclimated group. They were housed in chamber B where the ambient temperature, Ta, was controlled as $19 \pm 7 \mathrm{C}$ for the first 10 days, then increased to $33 \pm 7 \mathrm{C}$ for the next 14 days.

The second part of the experiment occurred more than one month later. Both sets of steers were placed in the field under identical conditions and data was recorded for 14 days.

During this experiment, $\mathrm{T}_{\mathrm{a}}$ was recorded every five minutes and the body core temperature, $\mathrm{T}_{\mathrm{b}}$, for each steer was recorded every eight minutes. For the purposes of biological research and model building, both $\mathrm{T}_{\mathrm{a}}$ and $\mathrm{T}_{\mathrm{b}}$ are hourly averaged.

The field data are used for our study of acclimation. In order to detect acclimation, the animal must be heat-stressed in the field. As luck would have it, day 13 is one of the very few days when $\mathrm{T}_{\mathrm{a}}$ was greater than $30^{\circ} \mathrm{C}$ (Figure 2). In figure 2 , the horizontal line indicates Ta is $30^{\circ} \mathrm{C}$. Also, of all the heat stress days, day 13 had the least number of missing observations. So, the data for the $13^{\text {th }}$ day was selected to test for the effect of acclimation.

\section{Data Analysis and Experimental Results}

\section{4.i. Univariate approach}

In this analysis, each steer is modeled separately and univariate analysis of variance is used to test the differences between treatments for each set of parameters (Seber and Wild, 1989).

To estimate the fixed parameters, a single nonlinear model is fit for each steer using Proc Nlin, and the parameter estimates for the individual animal are recorded. The model equation is:

$$
\mathrm{Y}_{\mathrm{tsx}}=\mathrm{f}\left(\mathrm{x} ; \kappa_{\mathrm{ts}}, \delta_{\mathrm{ts}}, \gamma_{\mathrm{ts}}, \mathrm{T}_{\mathrm{b} 0 \mathrm{ts}}\right)+\varepsilon_{\mathrm{tsx}}
$$

$\mathrm{Y}_{\mathrm{tsx}}$ is the body temperature for $\mathrm{s}^{\text {th }}$ steer in $\mathrm{t}^{\text {th }}$ treatment at time $\mathrm{x}$. Here, time, the independent variable is called $x$. The parameters for $s^{\text {th }}$ steer in $t^{\text {th }}$ treatment are $\kappa_{t s} \delta_{t s} \gamma_{t s}$ and $T_{b 0 t s}$. The mean, $\mathrm{f}\left(\mathrm{x} ; \mathrm{K}_{\mathrm{ts}}, \delta_{\mathrm{ts}}, \gamma_{\mathrm{ts}}, \mathrm{T}_{\mathrm{b} 0 \mathrm{ts}}\right)$ is the extended-PET-period-two model(Equation II). The distribution for $\mathrm{Y}_{\mathrm{tsx}}$ is: $Y_{t s x} \sim \operatorname{iidN}\left(f\left(x ; \kappa_{t s}, \delta_{t s}, \gamma_{t s}, T_{b(t) t s}\right), \sigma_{e}^{2}\right)$.

Next, a univariate analysis is performed on each parameter using proc mixed. The model for the parameters is:

$$
\mathrm{P}_{\mathrm{ts}}=\mathrm{u}+\operatorname{trt}_{\mathrm{l}}+\varepsilon_{\mathrm{r}} \text {. }
$$


$\mathrm{P}_{\mathrm{ts}}$ is the value of parameter $\mathrm{P}$ (one of $\kappa, \delta, \gamma, \mathrm{T}_{\mathrm{b} 0}$ ) for the $\mathrm{s}^{\text {th }}$ steer under $\mathrm{t}^{\text {th }}$ treatment, $\mathrm{u}$ is the overall mean, $\operatorname{trt}_{\mathrm{t}}$ is the effect caused by treatment $\mathrm{t}$, and $\mathrm{P}_{\mathrm{ts}} \sim \mathrm{iidN}\left(\mathrm{u}+\mathrm{trt}_{\mathrm{t}}, \sigma_{\mathrm{e}}{ }^{2}\right)$.

The results from the univariate analysis are shown in table 1 . The univariate approach does not detect a difference in any of the parameters; so, there is no indication of acclimation.

\section{4.ii. Nonlinear fixed model approach}

Unlike the univariate analysis, the steers under the same treatment are considered to be samples from the same population by nonlinear fixed model analysis. The extra-sum-of-squares test is used to detect treatment effects in the parameters based on significant difference between the full and partial models (Bates and Watts, 1988).

First, fit full model for all steers by proc Nlin, the full model equation is:

$$
Y_{t s x}=f\left(x ; \kappa_{t}, \delta_{t}, \gamma_{t}, T_{b 0 t}\right)+\varepsilon_{t s x}
$$

$\mathrm{Y}_{\mathrm{tsx}}$ is the body temperature for $\mathrm{s}^{\text {th }}$ steer in $\mathrm{t}^{\text {th }}$ treatment at time $\mathrm{x}$. Time, the independent variable is called $x$. The parameters for steers in $t^{\text {th }}$ treatment are $\kappa_{t} \delta_{t} \gamma_{t}$ and $T_{b 0 t}$. The mean, $\mathrm{f}\left(\mathrm{x} ; \kappa_{\mathrm{ts}}, \delta_{\mathrm{t}}, \gamma_{\mathrm{t}}, \mathrm{T}_{\mathrm{b} 0 \mathrm{t}}\right)$ is the extended-PET-period-two model(Equation II). The distribution for $\mathrm{Y}_{\mathrm{tsx}}$ is: $\mathrm{Y}_{\mathrm{tsx}} \sim \operatorname{iidN}\left(\mathrm{f}\left(\mathrm{x} ; \kappa_{\mathrm{ts}}, \delta_{\mathrm{t}}, \gamma_{\mathrm{t}}, \mathrm{T}_{\mathrm{b} 0 \mathrm{t}}\right), \sigma_{\mathrm{e}}^{2}\right)$.

Second, fit partial models for all steers using proc Nlin. The partial model is a nested model from the full model. The equations are:

$$
\begin{array}{ll}
\text { Partial Model 1: } & \mathrm{Y}_{\mathrm{tsx}}=\mathrm{f}\left(\mathrm{x} ; \kappa, \delta_{\mathrm{t}}, \gamma_{\mathrm{t}}, \mathrm{T}_{\mathrm{b} 0 \mathrm{t}}\right)+\varepsilon_{\mathrm{tsx}}, \\
\text { Partial Model 2: } & \mathrm{Y}_{\mathrm{tsx}}=\mathrm{f}\left(\mathrm{x} ; \kappa_{\mathrm{t}}, \delta, \gamma_{\mathrm{t}}, \mathrm{T}_{\mathrm{b} 0 \mathrm{t}}\right)+\varepsilon_{\mathrm{tsx}}, \\
\text { Partial Model 3: } & \mathrm{Y}_{\mathrm{tsx}}=\mathrm{f}\left(\mathrm{x} ; \kappa_{\mathrm{t}}, \delta_{\mathrm{t}}, \gamma, \mathrm{T}_{\mathrm{b} 0 \mathrm{t}}\right)+\varepsilon_{\mathrm{tsx}}, \\
\text { Partial Model 4: } & \mathrm{Y}_{\mathrm{tsx}}=\mathrm{f}\left(\mathrm{x} ; \kappa_{\mathrm{t}}, \delta_{\mathrm{t}}, \gamma_{\mathrm{t}}, \mathbf{T}_{\mathrm{b} \mathbf{0}}\right)+\varepsilon_{\mathrm{tsx}}
\end{array}
$$

Finally, the extra-sum-of-squares test is conducted to evaluate whether the reduction in sumof-squares for error is significant when the model is more complicated (D.M. Bates and D.L. Watts, 1988).

$\begin{array}{lclll}\text { Sources } & \text { SS } & \text { DF } & \text { Mean Square } & \text { F ratio } \\ \text { Extra parms } & \text { SSe=SSp-SSf } & \text { dfe=Pf-Pp } & \text { MSe=SSe/dfe } & \text { MSe/MSf } \\ \text { Full Model } & \text { SSf } & \text { dff }=\text { N-Pf } & \text { MSf=SSf/dff } & \\ \text { Partial Model } & \text { SSp } & \text { dfp=N-Pp } & & \end{array}$

This test is performed for each parameter. The null hypothesis that there is no difference between the full model and the partial model translates into a test for no difference among the treatment effects. 
The results from the nonlinear fixed model analysis are shown in table 2 . The nonlinear fixed approach did not detect any difference between treatments for any of the parameters. Once again, there is no indication of acclimation.

\section{4.iii. Nonlinear mixed model approach:}

Figure 3 shows the predicted $\mathrm{T}_{\mathrm{b}}$ curves for the steers from the population under treatment 1 (Control) and treatment 2(Potentially Acclimated). These plots show that although the steers come from the same population, the shapes of the predicted curves are quite different. So, the parameters for each steer are decomposed to two parts, the fixed part which is the population mean and the random part which is the variation among the population (Wolfinger, 2000). Specifically, the equation for this nonlinear mixed model is :

$$
Y_{t s x}=f\left(x ; \kappa_{t s}, \delta_{t s}, \gamma_{t s}, T_{b 0 t}\right)+\varepsilon_{t s x}
$$

Or: $\quad Y_{t s x}=f\left(x ; \kappa_{t}+b_{1 t s}, \delta_{t}+b_{2 t s}, \gamma_{t}+b_{3 t s}, T_{b 0 t}\right)+\varepsilon_{t s x}$

$\mathrm{Y}_{\mathrm{tsx}}$ is the body temperature for $\mathrm{s}^{\text {th }}$ steer in $\mathrm{t}^{\text {th }}$ treatment at time $\mathrm{x}$. Time, the independent variable is called $x$. The fixed parameters for $s^{\text {th }}$ steer in $t^{\text {th }}$ treatment are $\kappa_{t}, \delta_{t}, \gamma_{t s}$, and $T_{b 0 t}$ while the random effects are $b_{1 t s}, b_{2 t s}$, and $b_{3 t s}$. The mean for the $s^{\text {th }}$ steer from the population given the $t^{\text {th }}$ treatment, $f\left(x, \kappa_{t}+b_{1 t s}, \delta_{t}+b_{2 t s}, \gamma_{t}+b_{3 t s}, T_{b 0 t}\right)$ is the extended-PET-period-two model(Equation II). The distributional assumptions are as follows:

(1) $\varepsilon_{\mathrm{tsx}} \sim \mathrm{iidN}\left(0, \sigma_{\mathrm{e}}^{2}\right)$

(2) $\quad\left(\kappa_{t s}, \delta_{t s}, \gamma_{t s}\right)^{T} \sim \operatorname{MVN}\left(\left(\kappa_{t}, \delta_{t}, \gamma_{t}\right)^{T},\left[\begin{array}{ccc}\sigma_{\kappa}{ }^{2} & 0 & 0 \\ 0 & \sigma_{\delta}{ }^{2} & \operatorname{Cov}(\delta, \gamma) \\ 0 & \operatorname{Cov}(\delta, \gamma) & \sigma_{\gamma}{ }^{2}\end{array}\right]\right)$

(3) $Y_{t s x} \mid b_{1 t s}, b_{2 t s}, b_{3 t s} \sim \operatorname{iidN}\left(f\left(x, \kappa_{t}+b_{1 t s}, \delta_{1}+b_{2 t s}, \gamma_{t}+b_{3 t s}, T_{b 0 t}\right), \sigma_{e}^{2}\right)$

The covariance structure selected was based on our experience using the PET model with this type of data. Previous results showed a reoccurring pattern of minimal covariance between $K$ and the other two parameters, while there are a large negative covariance between $\delta$ and $\gamma$.

To detect treatment differences, fit the model for all steers using proc nlmixed, and get the two sets of parameter estimates with respect to the two treatments. The "contrast" or "estimate" statement may be used to test for differences in the parameter estimates between the two treatment populations.

The results from the nonlinear mixed model analysis are listed in table 3 . Since the random effect of an individual steer within the same treatment is concerned, the estimates for $\tau$ and $\kappa$ are different from those obtained by univariate and nonlinear fixed approach. In addition. table 3 shows there is a significant difference between treatments for parameters $\kappa(p=0.0009)$, $\delta(p=0.057)$, and $\tau(p=0.0001)$. Based on this result. acclimation is present. Examination of the 
parameters indicates steers will be quicker to adjust to heat-stress in the field, if they were exposed to a controlled heat-stressed environment beforehand. This management practice produces environmental acclimation.

\section{Simulation Studies}

As stated previously, the univariate analysis is recommended historically; the nonlinear fixed model analysis is potentially useful; and the nonlinear mixed model analysis is the newest approach. So we are eager to compare and contrast these three approaches. Simulations were used to investigate the behavior of the parameter estimates for each approach under five levels of random variation around the thermal constant $\kappa$. We want to know if the performance of one approach is better than the rest or if the performance varies with experimental conditions?

\section{5.i. Design}

The treatments are assumed to be $\tau_{1}=2$ and $\tau_{2}=1$, or equivalently, $\mathrm{k}_{1}=0.45$ and $\mathrm{k}_{2}=0.97$. Such values could indicate a biologically meaningful difference. Next, $\kappa_{1}$ and $\kappa_{2}$ are simulated using five common thermal constant variance levels $\left(\sigma_{\kappa}{ }^{2}=0.15,0.10,0.05,0.01\right.$ and 0.005$)$. The $\mathrm{T}_{\mathrm{a}}$ is simulated using parameter estimates from the experimental data, day 13 for a 2-period sinusoidal function: $\mathrm{u}_{\mathrm{a}}=30, \mathrm{a}_{1}=6.915, \mathrm{a}_{2}=-0.546, \phi_{1}=10.148, \phi_{2}=5.7$ (Equation I). The parameter values used to simulate $\mathrm{T}_{\mathrm{b}}$ come from the nonlinear mixed approach to the extended-PET-period-two model (Equation II), specifically, $\sigma_{\mathrm{e}}{ }^{2}=0.06, \sigma_{\delta}{ }^{2}=1.2, \sigma_{\gamma}{ }^{2}=0.001, \operatorname{cov}(\delta, \gamma)=-0.04, \mathrm{k}_{1}=0.45$, $\mathrm{k}_{2}=0.97, \mathrm{~d}_{1}=34.46, \mathrm{~d}_{2}=33.11, \mathrm{~g}_{1}=0.16, \mathrm{~g}_{2}=0.197, \mathrm{~T}_{\text {init }}=37.95, \mathrm{~T}_{\text {init } 2}=38.3$.

The data for 12 steers are simulated, six steers from each treatment population. For each of the five thermal constant variances levels, 500 sets of data were generated.

\section{5.ii. Analysis}

The analyses for the simulated data are exactly the same as the three approaches used for the experimental data described in part 4 . The methods are: the univariate approach that fits individual models to each steer (4.i), the nonlinear fixed approach that fits common full model and partial models for all the steers from the same population (4.ii), and the nonlinear mixed approach that takes into account the variation within the population (4.iii). All three approaches are applied to each set of data generated. Since $\kappa_{1}$ and $\kappa_{2}$ (or $\tau_{1}$ and $\tau_{2}$ ) are used to measure acclimation, for each approach and set of simulated data, the parameter estimates, the standard errors, the $95 \%$ confidence intervals of $\kappa_{1}$ and $\kappa_{2}$ (or $\tau_{1}$ and $\tau_{2}$ ), as well as, the p-values used to test for differences between the parameters are obtained.

\section{5.iii. Evaluation}

In order to compare the three approaches, we calculated the mean and standard error of the parameter estimates $\kappa_{1}, \kappa_{2}$, and their estimated difference, $\kappa_{1}-\kappa_{2}$. Additionally, the distributions of the estimates were checked for normality, bias, and skewness. The $95 \%$ confidence intervals about $\kappa_{1}-\kappa_{2}$ and the powers of the difference were calculated to compare the performance for inference and hypothesis testing. 
The bias for parameters is the difference between the estimate and the true value of parameter. The acceptable percentage of no nonlinear behavior is 1 (Bates and Watts, 1988). Also, we applied t-tests to test if the bias was significant different from 0 or not.

The shapes of the distributions were compared for normality and skewness. Proc univariate was used to produce the p-values for Shapiro-Wilk test of normality, as well as, the skewness values to measure departure from symmetry.

The $95 \%$ confidence intervals of the difference $\kappa_{1}-\kappa_{2}$ were examined to find the percentage of intervals that cover the true difference of -0.52 .

Finally for each approach, we calculated the power of the test for treatment differences. Power, the probability rejecting a false null hypothesis, given $\alpha=0.05$, is obtained by the rejection rate for the null hypothesis that $\mathrm{k}_{1}=\mathrm{k}_{2}$, or equivalently, $\tau_{1}=\tau_{2}$.

\section{Simulation Results and Discussion}

\section{6.i. Parameter Estimation, Bias, Distribution and Skewness}

Tables from 4.a to 4.c show the means and the standard errors of $\kappa_{1}, \kappa_{2}$ and $\kappa_{1}-\kappa_{2}$ for the simulated data. Since the true values for $\kappa_{1}, \kappa_{2}$ and difference are $0.45,0.97$ and -0.52 respectively, the bias is obtained by comparing the estimates and true values.

To check the bias, we used the t-test with the null hypothesis: (a) For $\kappa_{1}, \mathrm{H}_{0}: \kappa_{1}=0.45$; (b) For $\kappa_{2}, H_{0}: \kappa_{2}=0.97$; (c) For $\kappa_{1}-\kappa_{2}, H_{0}: \kappa_{1}-\kappa_{2}=-0.52$. No bias was detected. None of the tests were significant(Results not shown). As far as the percent bias, $\kappa_{1}$ is overestimated by the univariate approach with the range around $3 \%$ when $\sigma_{\kappa}{ }^{2}<0.15$, while it is underestimated with the range between $11 \%$ and $33 \%$ by the nonlinear fixed and mixed approaches(Table 4.a). Similarly, $\kappa_{2}$ is overestimated by the univariate approach with the range around $6 \%$ when $\sigma_{\kappa}{ }^{2}<0.15$, while it is underestimated with the range between $6 \%$ and $12 \%$ by the nonlinear fixed and mixed approaches (Table 4.b). However, the bias percentage for the difference, $\kappa_{1}-\kappa_{2}$, is close to $1 \%$ when $\sigma_{\kappa}{ }^{2}>0.05$ for nonlinear mixed approach, but its range is between $8 \%$ and $10 \%$ for univariate approach and between $2 \%$ and $31 \%$ for the nonlinear fixed approach(Table 4.c).

Table 5 lists the p-values for checking the normality of the distribution of the difference, $\kappa_{1}-\kappa_{2}$. by the Shapiro-Wilk test. The tests indicate significant deviations from normality in all but a few nonlinear fixed cases.

For skewness, the closer the distribution is to normal, the closer the skewness is to zero. One is the practically accepted critical value (Bates and Watts, 1988). Results indicate that when variance is equal to or less than 0.01 , there is serious skewness for $\kappa_{1}, \kappa_{2}$ and $\kappa_{1}-\kappa_{2}$ by the nonlinear fixed approach (Table 6.b). Also, when the variance is 0.05 , the univariate approach has serious skewness on $\kappa_{1}$ (Table 6.a). The nonlinear mixed approach does not appear to be seriously skewed although for the difference, $\kappa_{1}-\kappa_{2}$, the univariate approach is less skewed when $\sigma_{\mathrm{k}}{ }^{2}<0.05$ (Table 6.c, Table 6.a).

\section{6.ii. 95\% Confidence Interval Coverage}

Table 7 indicates the nonlinear fixed model is so conservative that $99 \%$ of the $95 \%$ confidence inter als for $\kappa_{1}-\kappa_{2}$ cover the true value. When the variance, $\sigma_{k}{ }^{2}$. is less than 0.05 , the univariate approach is most efficient. This makes sense because when the variance is small, the steers' 
thermal constants can be regarded as homogeneous and the random effect among steers is negligible. On the other hand, when the common variance of $\kappa_{1}$ and $\kappa_{2}$ is more than 0.05 , the nonlinear mixed model analysis is best and most reliable. This is reasonable because as the variance increases, the random effect becomes a more important component of the model.

\section{6.iii. Power to Detect Treatment Differences}

For the nonlinear mixed approach, the power decreases as the thermal variance decreases, although this approach generally has enough power to detect the treatment difference (power $>.82$ ) Table 8 . On the other hand, the power of the univariate approach is poor when variation among thermal constants, $\sigma_{\kappa}{ }^{2}$, is large $(>0.05)$. However, the power improves dramatically as the variance becomes smaller. Eventually, the univariate approach begins to out-perform the nonlinear mixed model approach. Finally, the nonlinear fixed model approach is the least powerful of the three approaches for the variance levels studied.

\section{Summary}

The extended-PET-period-two model is a useful tool for studying heat-stress behavior in steers. The model not only gives biologically meaningful parameters but also enables investigators to test for significant treatment differences in populations. In the case of environmental acclimation, the model can be used to analyze the behavior of steers that are thermally challenged in the field and check for differences between steers previously exposed to controlled heat stress conditions and those who were not.

The simulation study showed that the performance of the three approaches (univariate, nonlinear fixed and nonlinear mixed) used to evaluate the treatments (thermoneutral, potentially acclimated) depends on the experimental conditions. The nonlinear mixed model is recommended when the random variation within thermal constants among subjects (steers) is at least 0.05 . The traditional univariate approach is preferred when the thermal constants are more homogeneous. The most promising use of the nonlinear fixed model is to provide parameters for the univariate approach and initial values for the nonlinear mixed approach. Experience shows that convergence appears to be less an issue when the random effects are omitted and proc Nlin is used for obtaining the initial value estimates.

For the motivating study - the acclimation experiment, the three approaches lead to different conclusions. Since the variation among thermal constants (lags) of the steers is approximately 0.15 , the simulation study indicates the nonlinear mixed model is the best approach. The resulting analysis demonstrates environmental acclimation. It indicates that the steers are quicker to adjust to heat-stress in the field, if they are previously exposed to a controlled hot chamber temperature. Thus, environmental acclimation is possible and management practices based on this concept are worthy of further study. 


\section{References}

Bates, D.M. and Watts, D.L. 1988. Nonlinear Regression Analysis \& Its Applications . John Wiley \& Sons, New York.

Feng, Sheng, Anne Parkhurst, Kent Eskridge, Daryl Travnicek, Donald Spiers, G. L. Hahn, and Terry Mader, 2001. "Assessing Refinements In Modeling Sinusoidal Conditions Used to Drive Cattle Body Temperatures". Proceedings of the $13^{\text {th }}$ Kansas State University Conference.

Hahn, G.L. 1989. "Body temperature rhythms in farm animals". Proceedings of $11^{\text {th }}$ ISBCongress. SPB Academic Publishing bv, The Hage, The Netherlands. 325-337.

Parkhurst, A.M., Eskridge, K.M., and Travnicek, D.A. 2000. "Evaluating nonlinear mixed model methods for fitting Newton's law of cooling with a sinusoidal forcing function".

Parkhurst, A.M., G.L.Hahn, K.M.Eskridge, D.A.Travnicek, and H.D.Liu. 1999. "Predicting body temperature of cattle during stages of exposure to controlled hot cyclic air temperature using metrics with specified delays", Proceedings of the International Congress of Biometeorology \& International Conference on Urban Climatology Nov 8-12 p 274-280.

Seber ,G.A.F and Wild,C.J. 1989. Nonlinear Regression. John Wiley \& Sons, New York. Wolfinger, Russ. 2000. "Fitting Nonlinear and Generalized Mixed Models with PROC NLMIXED”. 2000 Joint Statistics Meetings Continuing Education Series. 
Table $1 \quad$ Univariate Analysis of Parameter Differences

\begin{tabular}{|c|c|c|c|}
\hline \multirow{2}{*}{ Parameters } & \multicolumn{2}{|c|}{ Parameter means(standard error) } & \multirow{2}{*}{ P-value } \\
\cline { 2 - 3 } & Control & Acclimated & 0.276 \\
\hline$\tau$ & $1.22(0.569)$ & $0.92(0.271)$ & 0.396 \\
\hline$\kappa$ & $0.94(0.420)$ & $1.13(0.289)$ & 0.948 \\
\hline$T_{\text {Binitial }}$ & $38.33(0.401)$ & $38.31(0.558)$ & 0.666 \\
\hline$\delta$ & $33.40(1.239)$ & $33.11(0.964)$ & 0.707 \\
\hline$\gamma$ & $0.187(0.049)$ & $0.197(0.035)$ & \\
\hline
\end{tabular}

Table $2 \quad$ Nonlinear Fixed Model Analysis of Parameter Differences

\begin{tabular}{|c|c|c|c|}
\hline \multirow{2}{*}{ Parameters } & \multicolumn{2}{|c|}{ Parameter means } & \multirow{2}{*}{ P-value } \\
\cline { 2 - 3 } & Control & Acclimated & 0.452 \\
\hline$\tau$ & $1.12(0.194)$ & $0.92(0.172)$ & 0.458 \\
\hline$\kappa$ & $0.87(0.173)$ & $1.06(0.227)$ & 0.944 \\
\hline $\mathrm{T}_{\text {Binitial }}$ & $38.31(0.502)$ & $38.26(0.619)$ & 0.433 \\
\hline$\delta$ & $33.42(0.309)$ & $33.08(0.298)$ & 0.407 \\
\hline$\gamma$ & $0.187(0.010)$ & $0.198(0.009)$ & \\
\hline
\end{tabular}

Table 3 Nonlinear Mixed Model Analysis of Parameter Differences

\begin{tabular}{|c|c|c|c|}
\hline \multirow{2}{*}{ Parameters } & \multicolumn{2}{|c|}{ Parameter means } & \multirow{2}{*}{ P-value } \\
\cline { 2 - 3 } & Control & Acclimated & 0.0001 \\
\hline$\tau$ & $2.616(0.262)$ & $0.844(0.119)$ & 0.0009 \\
\hline$\kappa$ & $0.321(0.045)$ & $1.166(0.171)$ & 0.332 \\
\hline $\mathrm{T}_{\text {Binitial }}$ & $37.95(0.184)$ & $38.29(0.284)$ & 0.057 \\
\hline$\delta$ & $34.46(0.422)$ & $33.11(0.468)$ & 0.134 \\
\hline$\gamma$ & $0.156(0.018)$ & $0.196(0.016)$ & \\
\hline
\end{tabular}

Table 4.a Estimated Means, (SE), and Percent Bias for True Kappal of 0.45 (500 Simulations)

\begin{tabular}{|c|c|c|c|c|}
\hline True & True & \multicolumn{3}{|c|}{ Approach } \\
\cline { 3 - 5 } $\operatorname{Var}(\kappa)$ & $\mathrm{SE}\left(\kappa_{1}-\kappa_{2}\right)$ & Univariate & Nlin Fixed Model & Nlin Mixed Model \\
\hline 0.15 & 0.548 & $0.332(0.651)-26.2$ & $0.399(0.101)-11.3$ & $0.386(0.110)-14.2$ \\
\hline 0.10 & 0.447 & $0.462(0.106) 2.7$ & $0.398(0.099)-11.6$ & $0.382(0.114)-15.1$ \\
\hline 0.05 & 0.316 & $0.464(0.105) 3.1$ & $0.378(0.106)-16.0$ & $0.358(0.108)-20.4$ \\
\hline 0.01 & 0.141 & $0.462(0.062) 2.7$ & $0.320(0.132)-28.9$ & $0.322(0.163)-28.4$ \\
\hline 0.005 & 0.100 & $0.463(0.054) 2.9$ & $0.304(0.150)-32.4$ & $0.359(0.208)-20.2$ \\
\hline
\end{tabular}


Table 4.b Estimated Means, (SE), and Percent Bias for True Kappa2 of 0.97 (500 Simulations)

\begin{tabular}{|c|c|c|c|c|}
\hline True & True & \multicolumn{3}{|c|}{ Approach } \\
\cline { 3 - 5 } $\operatorname{Var}(\kappa)$ & $\mathrm{SE}\left(\kappa_{1}-\kappa_{2}\right)$ & Univariate & Nlin Fixed Model & Nlin Mixed Model \\
\hline 0.15 & 0.548 & $0.447(0.713)-53.9$ & $0.906(0.127)-6.6$ & $0.906(0.131)-6.6$ \\
\hline 0.10 & 0.447 & $1.036(0.207) 6.8$ & $0.899(0.130)-7.3$ & $0.902(0.132)-7.0$ \\
\hline 0.05 & 0.316 & $1.030(0.173) 6.2$ & $0.902(0.132)-7.0$ & $0.897(0.135)-7.5$ \\
\hline 0.01 & 0.141 & $1.025(0.130) 5.7$ & $0.919(0.231)-5.3$ & $0.873(0.170)-10.0$ \\
\hline 0.005 & 0.100 & $1.020(0.127) 5.2$ & $0.982(0.325) 1.2$ & $0.881(0.213)-9.2$ \\
\hline
\end{tabular}

Table 4.c Estimated Means, (SE), and Percent Bias for True Kappa Difference of -0.52 (500 Simulations)

\begin{tabular}{|c|c|c|c|c|}
\hline True & True & \multicolumn{3}{|c|}{ Approach } \\
\cline { 3 - 5 } $\operatorname{Var}(\kappa)$ & $\mathrm{SE}\left(\kappa_{1}-\kappa_{2}\right)$ & Univariate & Nlin Fixed Model & Nlin Mixed Model \\
\hline 0.15 & 0.548 & $0.145(0.736)>100$ & $-0.506(0.155) 2.7$ & $-0.523(0.164)-0.6$ \\
\hline 0.10 & 0.447 & $-0.573(0.230)-10.2$ & $-0.501(0.161) 3.7$ & $-0.519(0.167) 0.2$ \\
\hline 0.05 & 0.316 & $-0.566(0.204)-8.8$ & $-0.524(0.157)-0.8$ & $-0.539(0.166)-3.7$ \\
\hline 0.01 & 0.141 & $-0.563(0.146)-8.3$ & $-0.600(0.270)-15.4$ & $-0.552(0.231)-6.2$ \\
\hline 0.005 & 0.100 & $-0.563(0.139)-8.3$ & $-0.678(0.388)-30.4$ & $-0.522(0.321)-0.4$ \\
\hline
\end{tabular}

Table 5 Normality Test p-values for Difference Variable Kappa1-Kappa2(500 Simulations)

\begin{tabular}{|c|c|c|c|}
\hline \multirow{2}{*}{ True Var(K) } & \multicolumn{3}{|c|}{ Approach } \\
\cline { 2 - 4 } & Univariate & Nlin Fixed Model & Nlin Mixed Model \\
\hline 0.15 & $<0.0001$ & 0.0946 & 0.0015 \\
\hline 0.10 & $<0.0001$ & 0.401 & 0.0610 \\
\hline 0.05 & $<0.0001$ & 0.137 & 0.0562 \\
\hline 0.01 & $<0.0001$ & $<0.0001$ & 0.0618 \\
\hline 0.005 & $<0.0001$ & $<0.0001$ & 0.0009 \\
\hline
\end{tabular}

Table 6.a Skewness for Kappal (500 Simulations)

\begin{tabular}{|c|c|c|c|}
\hline \multirow{2}{*}{ True Var( $)$} & \multicolumn{3}{|c|}{ Approach } \\
\cline { 2 - 4 } & Univariate & Nlin Fixed Model & Nlin Mixed Model \\
\hline 0.15 & -0.497 & 0.273 & 0.390 \\
\hline 0.10 & 0.358 & 0.327 & 0.298 \\
\hline 0.05 & 1.848 & 0.478 & 0.327 \\
\hline 0.01 & 0.862 & 1.061 & 0.842 \\
\hline 0.005 & 0.946 & 1.093 & 0.774 \\
\hline
\end{tabular}


Table 6.b Skewness for Kappa2 (500 Simulations)

\begin{tabular}{|c|c|c|c|}
\hline \multirow{2}{*}{ True Var $(\kappa)$} & \multicolumn{3}{|c|}{ Approach } \\
\cline { 2 - 4 } & Univariate & Nlin Fixed Model & Nlin Mixed Model \\
\hline 0.15 & -0.510 & 0.459 & 0.621 \\
\hline 0.10 & 0.861 & 0.408 & 0.593 \\
\hline 0.05 & 0.986 & 0.540 & 0.490 \\
\hline 0.01 & 0.850 & 1.69 & 0.826 \\
\hline 0.005 & 0.841 & 1.983 & 0.988 \\
\hline
\end{tabular}

Table 6.c Skewness for Difference Variable (Kappa1-Kappa2) (500 Simulations)

\begin{tabular}{|c|c|c|c|}
\hline \multirow{2}{*}{ True Var(K) } & \multicolumn{3}{|c|}{ Approach } \\
\cline { 2 - 4 } & Univariate & Nlin Fixed Model & Nlin Mixed Model \\
\hline 0.15 & -0.035 & -0.216 & 0.621 \\
\hline 0.10 & -0.738 & -0.066 & 0.593 \\
\hline 0.05 & -0.296 & -0.226 & 0.490 \\
\hline 0.01 & -0.492 & -1.087 & 0.826 \\
\hline 0.005 & -0.530 & -1.312 & 0.988 \\
\hline
\end{tabular}

Table 7 Coverage of 95\% Confidence Interval for kappal-kappa2 (500 simulations)

\begin{tabular}{|c|c|c|c|}
\hline \multirow{2}{*}{ True Var( $\mathrm{K})$} & \multicolumn{3}{|c|}{ Approach } \\
\cline { 2 - 4 } & Univariate & Nlin Fixed Model & Nlin Mixed Model \\
\hline 0.15 & 0.236 & 0.992 & 0.888 \\
\hline 0.10 & 0.645 & 0.994 & 0.856 \\
\hline 0.05 & 0.792 & 0.992 & 0.832 \\
\hline 0.01 & 0.934 & 0.988 & 0.583 \\
\hline 0.005 & 0.9398 & 0.99 & 0.389 \\
\hline
\end{tabular}

Table 8 Simulated Power for Difference of One in Lag (500 simulations)

\begin{tabular}{|c|c|c|c|}
\hline \multirow{2}{*}{ True Var( $(\kappa)$} & \multicolumn{3}{|c|}{ Approach } \\
\cline { 2 - 4 } & Univariate & Nlin Fixed Model & Nlin Mixed Model \\
\hline 0.15 & 0.268 & 0.258 & 0.959 \\
\hline 0.10 & 0.665 & 0.264 & 0.954 \\
\hline 0.05 & 0.824 & 0.30 & 0.966 \\
\hline 0.01 & 0.976 & 0.456 & 0.939 \\
\hline 0.005 & 0.984 & 0.472 & 0.839 \\
\hline
\end{tabular}




\section{Applied Statistics in Agriculture}

Figure 1. $\operatorname{Lag}(\tau)$ between $\operatorname{Body}\left(\mathrm{T}_{\mathrm{b}}\right)$ and $\operatorname{Air} \operatorname{Temp}\left(\mathrm{T}_{\mathrm{a}}\right)$

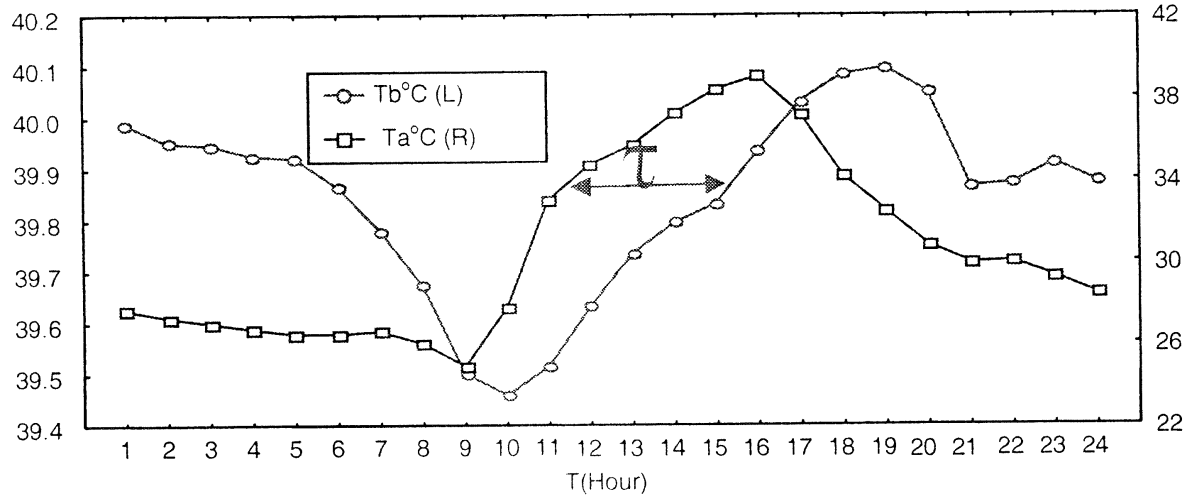

Figure 2. Air $\operatorname{Temp}\left(\mathrm{T}_{\mathrm{a}}{ }^{\circ} \mathrm{C}\right)$ Exceeding Bar Indicates the Heat-Stress Days
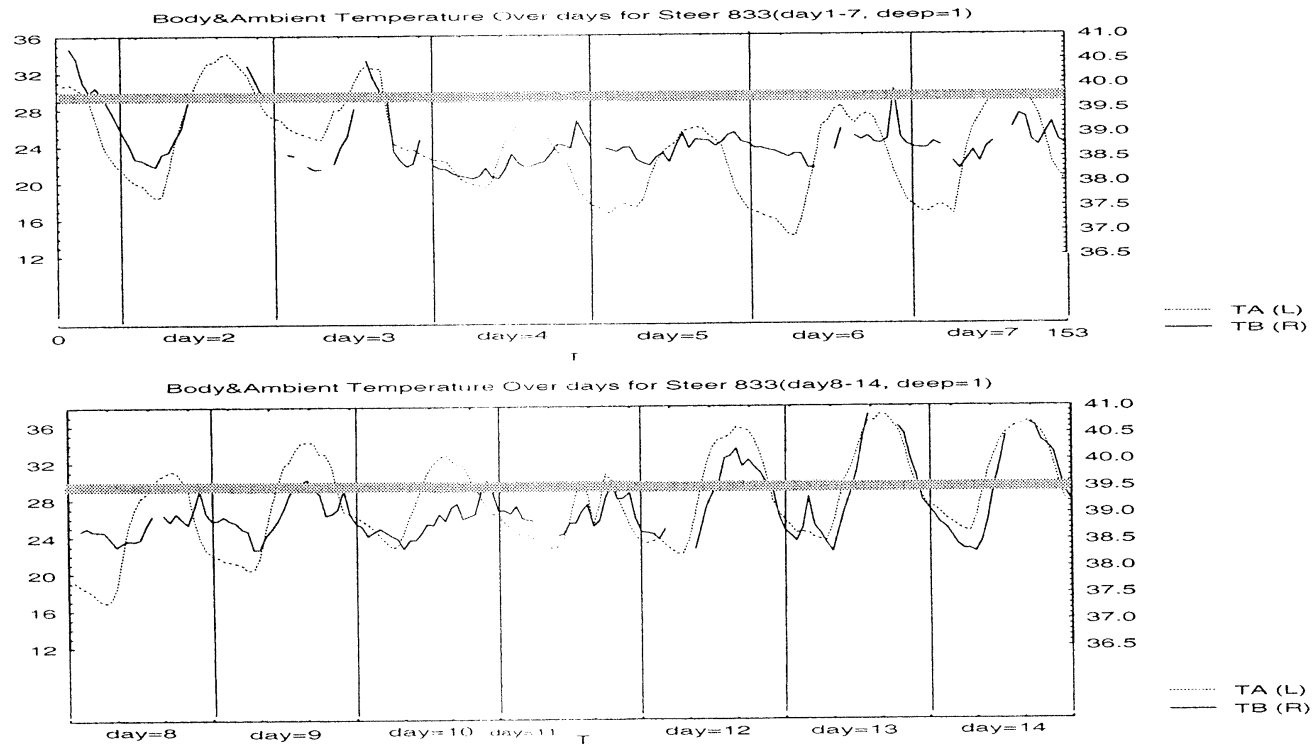

Figure 3. Predicted $T_{b}$ vs Ta Curves for Steers from Controlled and Acclimated Populations
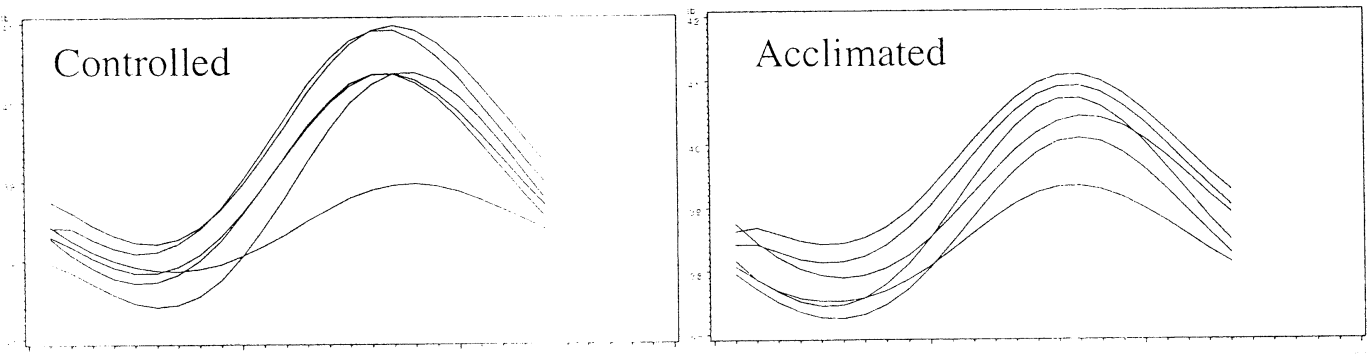


\section{Appendix 1. SAS Code for univariate approach}

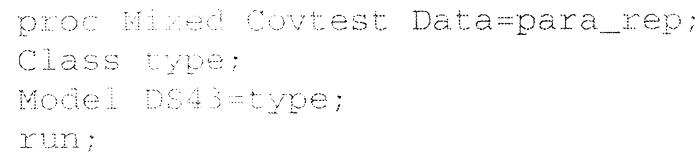

\section{Appendix 2. SAS Code for nonlinear fixed approach}

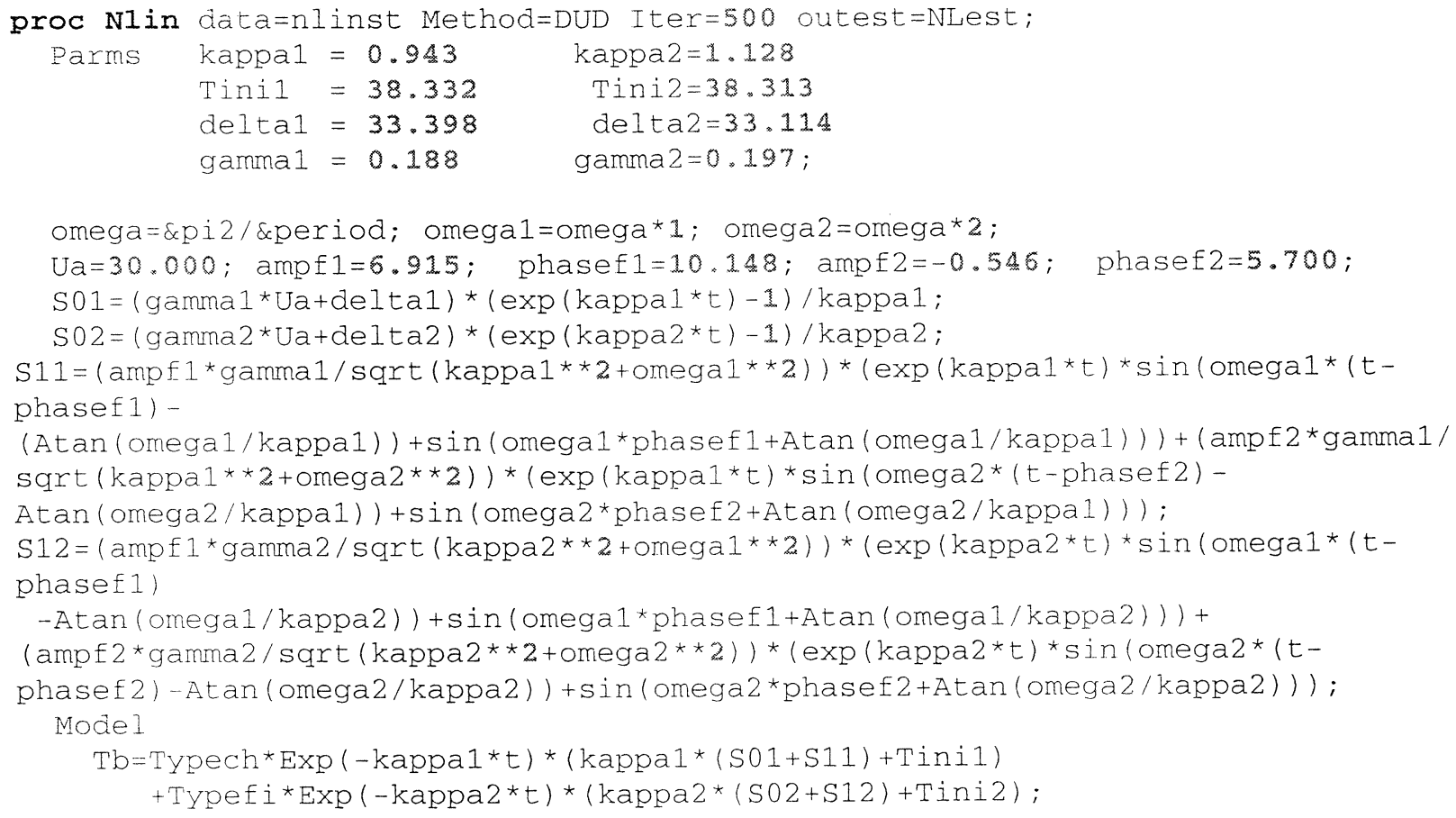

run;

Appendix 3. SAS Code for nonlinear mixed approach

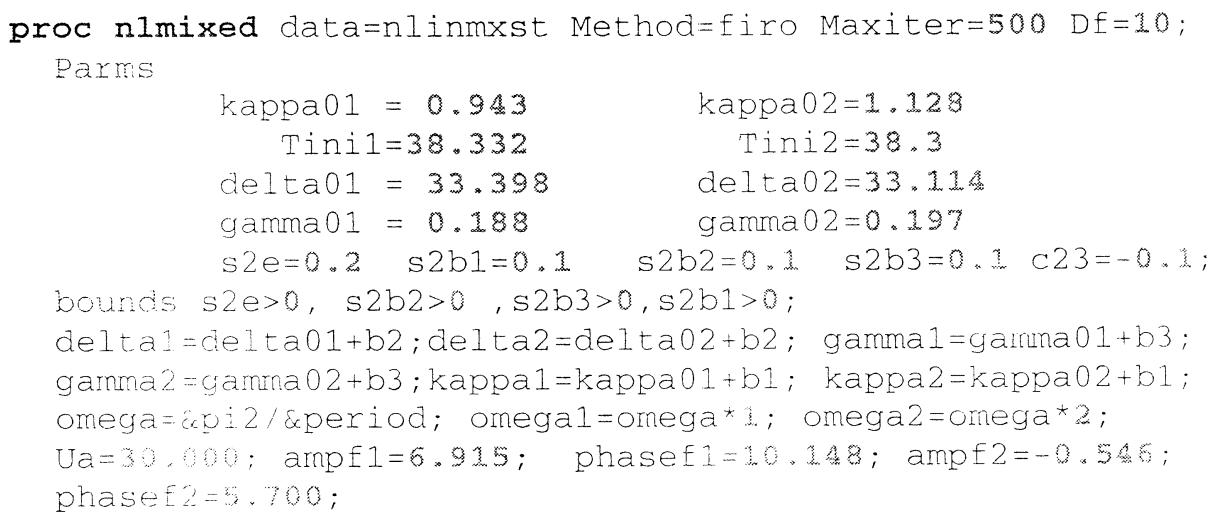




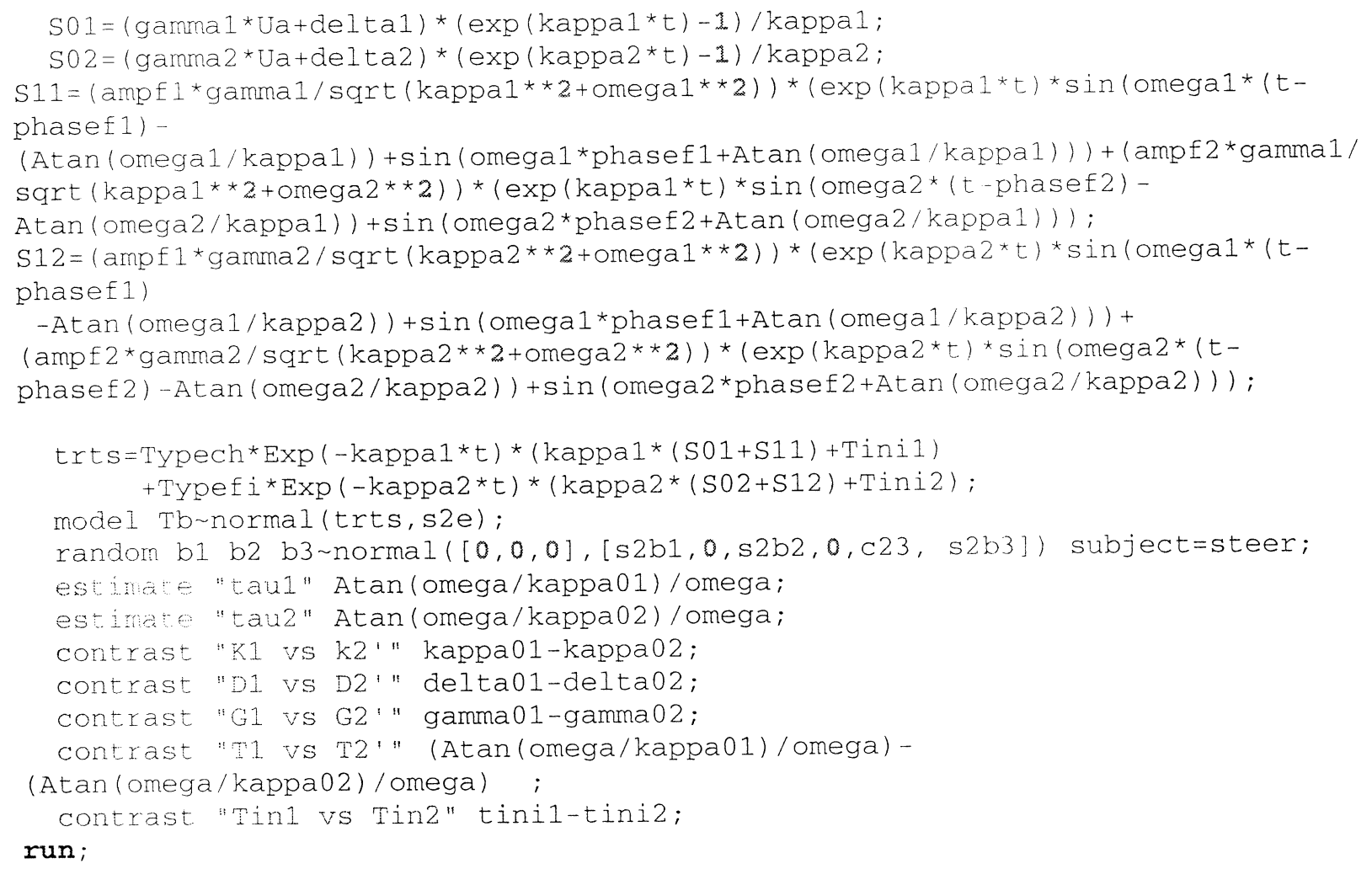

